\title{
In situ measurement and closed-loop control for powder supply processes
}

\author{
Retrofittable solution in the context of laser metal deposition
}

\author{
Philipp Peter Breese ${ }^{1,2}$ (D) . Tobias Hauser ${ }^{2,3} \cdot$ Daniel Regulin ${ }^{2} \cdot$ Stefan Seebauer ${ }^{2,4} \cdot$ Christian Rupprecht $^{1}$
}

Received: 28 January 2021 / Accepted: 7 June 2021 / Published online: 24 June 2021

(C) The Author(s) 2021

\begin{abstract}
The powder mass flow rate is one of the main parameters regarding the geometrical precision of built components in the additive manufacturing process of laser metal deposition. However, its accuracy, constancy, and repeatability over the course of the running process is not given. Reasons among others are the performance of the powder conveyors, the complex nature of the powder behavior, and the resulting issues with existing closed-loop control approaches. Additionally, a direct in situ measurement of the powder mass flow rate is only possible with intrusive methods. This publication introduces a novel approach to measure the current powder mass flow rate at a frequency of $125 \mathrm{~Hz}$. The volumetric powder flow evaluation given by a simple optical sensor concept was transferred to a mass flow rate through mathematical dependencies. They were found experimentally for a nickel-based powder (Inconel 625) and are valid for a wide range of mass flow rates. With this, the dynamic behavior of a vibration powder feeder was investigated and a memory effect dependent on previous powder feeder speeds was discovered. Next, a closed-loop control with the received sensor signal was implemented. The concept as a whole gives a repeatable and accurate powder mass flow rate while being universally retrofittable and applicable. In a final step, the improved dynamic and steady performance of the powder mass flow rate with closed-loop control was validated. It showed a reduction of mean relative errors for step responses of up to $81 \%$ compared to the uncontrolled cases.
\end{abstract}

Keywords Additive manufacturing $\cdot$ Laser metal deposition $\cdot$ Powder $\cdot$ Powder measurement $\cdot$ Mass flow rate

\section{Introduction and problem statement}

For the additive manufacturing (AM) process of laser metal deposition (LMD), metal powder is injected pneumatically into the process chamber. A laser is used as heat source to melt the powder and to create a melt pool. As the

Philipp Peter Breese

philipp.p.breese@ campus.tu-berlin.de

Tobias Hauser

tobias.hauser@siemens.com

1 Technische Universität Berlin, Coating Technology, Straße des 17. Juni 135, 10623 Berlin, Germany

2 Siemens AG, Otto-Hahn-Ring 6, 81739 Munich, Germany

3 Department of Engineering Sciences and Mathematics, Luleå University of Technology, S-971 87, Luleå, Sweden

4 Institute of Solid State Physics, TU Vienna, Wiedner Hauptstr. 8-10, 1040 Vienna, Austria injection nozzle moves relatively to the substrate, material is deposited in this way. The manufacturing process is realized in layers and the intended component is build up. Common other names for the same AM process are laser cladding, laser engineering net shape (LENS), or direct metal deposition (DMD).

The geometry of the manufactured component is determined by three main parameters: the laser power, the travel speed of the nozzle, and the powder mass flow rate $\dot{m}[1,2]$. With a precise and repeatable selection of powder mass flow rates, the LMD process as a whole is improved. In the first place, the geometry accuracy of the manufactured LMD components can be increased as shown by [3]. Mahamood and Akinlabi [4] also showed for a titanium alloy the influence of the powder mass flow rate on other component parameters: the microhardness and the surface roughness increase with increasing powder mass flow rates. With a constant, non-fluctuating mass flow rate, these parameters are kept constant over the component as well. Furthermore, special features like material transitions 
by in-process powder mixing are possible as demonstrated by [5]. A usage in powder-based coating processes like thermal barrier coatings (TBC) is also possible. Reason for this is that the working principles are the same and the utilized hardware, especially the powder conveyor is often identical.

However, Yan [6] showed that only a limited number of direct in situ measurement principles is available. They require an interference with the powder flow itself like inserting heat or charging the particles which marks an intrusive measurement [6]. But the non-intrusive in-process tracing of the powder mass flow rate is important as the AM process should not be affected. Furthermore, the amount of powder is selected with the motor speed of the conveyors. Time-consuming and repetitive calibrations are therefore inevitable while precision and repeatability are not given per se.

An improvement is accomplished by the usage of closedloop control approaches for the powder mass flow rate. The goal is to achieve a constant powder mass flow rate over time with deviations as low as possible. Furthermore, overshoot of the rate should be prevented. The increased powder mass flow rate during overshoots not only has an influence on the manufactured geometries, but also brings an unnecessary rise in powder consumption. This requires more cleaning efforts of not processed powder in the process chamber and increases material costs.

\begin{tabular}{|c|c|c|}
\hline $\mathrm{AM}$ & Additive manufacturing & \\
\hline LMD & Laser metal deposition & \\
\hline$M R E$ & Mean relative error & $\%$ \\
\hline $\mathrm{NC}$ & Numerical control & \\
\hline PID & Proportional-integral-differential & \\
\hline$K_{P, I, D}$ & PID controller gains & \\
\hline$K_{S}$ & Stationary gain & \\
\hline$S$ & $\begin{array}{l}\text { Analog sensor value of the } \\
\text { powder flow measurement }\end{array}$ & $\mathrm{mV}$ \\
\hline$T_{\Sigma}$ & Sum time constant & $\mathrm{s}$ \\
\hline$\dot{V}$ & $\begin{array}{l}\text { Volume flow rate of argon } \\
\text { carrier gas }\end{array}$ & $1 / \min$ \\
\hline$a$ & $\begin{array}{l}\text { Scaling factor for sensor output } \\
\text { dependency function }\end{array}$ & $\begin{array}{l}{[(\mathrm{mV} \min } \\
\left.\mathrm{g})^{0.63}\right]\end{array}$ \\
\hline$b$ & $\begin{array}{l}\text { Exponent for sensor output } \\
\text { dependency function }\end{array}$ & {$[-]$} \\
\hline$d_{50}$ & Median powder particle size & $\mu \mathrm{m}$ \\
\hline$\dot{m}$ & Powder mass flow rate & $\mathrm{g} / \mathrm{min}$ \\
\hline$n_{\text {meas }}$ & $\begin{array}{l}\text { Number of measurements for } \\
\text { control validation test }\end{array}$ & {$[-]$} \\
\hline
\end{tabular}

One method to overcome the mentioned issues is the use of a gravimetric powder feeding system also called lossin-weight powder feeding [7]. The whole conveyor system is placed on a weighing scale or a load cell respectively. Over the course of the process, the amount of powder weight decreases and therefore the powder mass flow rate is tracked. With this information, a closed-loop control can be implemented that regulates the motor speed of the conveyor to maintain the selected mass flow rate [8]. This approach can bring a major improvement, but also has several disadvantages. The system is vulnerable to disturbances which directly affect the measurement of the powder weight. This includes external disturbances like pull on the connected tubes or vibrations near the conveyor, especially in an industrial environment. But also internal disturbances can play a role like sudden changes in pressure that cause inaccuracies in the precision weight measurement. The frequency of the mass flow rate determination can also be rather low so that the closed-loop control is not ideally performing. Furthermore, the system is expensive in general and a retrofit is costly or even not possible.

Due to this, other closed-loop methods for powder mass flow rate control exist. For example, Tang et al. [3] demonstrated a model approach for the powder transport and the conveyor motor itself to ensure constant mass flow rates. Another way is to use an indirect measurement as data basis for a closed-loop control, e.g., a pressure sensor as shown by [9]. Again, disadvantages cannot be ruled out for these like their retrofittability, costs, accuracy, dynamic behavior, or adaptability as they are too specific. Therefore, this publication introduces a novel approach based on an indirect in situ measurement with a robust optical sensor concept at low costs. The proof of concept for the determination of the actual powder mass flow rate was done with a mathematical model. The information gathered from this was utilized in a closed-loop control and detailed investigations on the conveyor behavior were conducted. In this way, a precise and repeatable powder mass flow rate at high adaptability becomes available.

\section{Experimental setup}

The experiments referred to in this publication were carried out in the laboratories of the Siemens AG in Munich, Germany. In the following section, the utilized equipment, devices, and sensors are presented with the reasons of their usage. An overview of the setup is given which is also depicted schematically in Fig. 1.

\subsection{Powder Feeder}

The powder was conveyed pneumatically to the process chamber with a powder feeder. In general, inert gas is used as carrier gas. It does not cause any additional unwanted effects in the LMD process like oxidation. In this case, 
Fig. 1 Schematic overview of the experimental setup consisting of vibration powder feeder, optical sensor, weighing scale and glass beaker, and numerical control (NC) and computer for data recording purposes

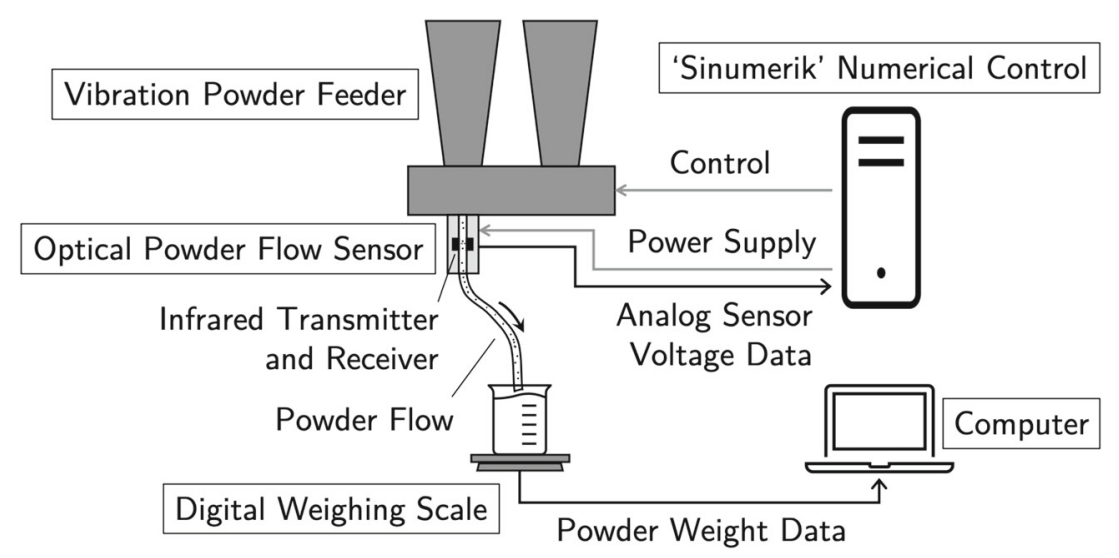

argon was used. The gas was pressurized for the transport and its volumetric amount $\dot{V}$ was set in $1 / \mathrm{m}$ at the feeder. The powder supply was only active when the gas flow was initialized and running. In this way, blockages were avoided as settling powder particles are less likely to occur with a constantly present gas flow. The volumetric carrier gas value was kept constant over the measurements regarding to the set target value. Typical values for the amount of carrier gas used in LMD processes are around 2 to $101 /$ min.

In general, two types of powder feeders are the most common: vibration feeders (also referred to as vibratory feeders or vibrating feeders) and disk feeders. For both, the powder for the supply is stored inside a sealed container as a part of the feeder which can be opened for refill purposes and dismounted for cleaning and switching powders.

The vibration feeder has a half-open, oscillating metal chute to convey the powder. With the oscillation, the powder is transported along over the chute and drops at its end into a small funnel and the following tube. Then, it is carried away with the carrier gas flow. New powder comes simultaneously from the container onto the chute to maintain the powder flow. The amount of conveyed powder is determined by the feeder speed which describes the working stroke of the steady rate oscillation.

In this publication, the vibration feeder Flowmotion by Medicoat was utilized. It has two separate conveyor lines and therefore two containers for powder storage in total, but only one of the lines was considered in the following. As mentioned before, its feeder speed is the working stroke level. It can be set in percent from 0 to $100 \%$ with the maximum being around $0.5 \mathrm{~mm}$. The frequency of the oscillation is determined at a constant rate of $109.3 \mathrm{~Hz}$ for the considered conveyor line.

The decision to use a vibration feeder and to focus on it had two reasons. Firstly, vibration feeders have a more dynamic behavior than disk feeders. Changes in feeder speed are realized faster for the vibration system due to its lower inertia compared to the rotating disk system.
This makes them more suitable for control applications. Secondly, vibration feeders are less precise and repeatable in their powder supply behavior. This gives a higher potential for improvements by implementation of a closed-loop control. Due to these two aspects, the focus was solely put on a vibration feeder.

\subsection{Powder parameters}

One of the central, most important aspects of the process to consider is the powder itself. A big variety of different alloys and compositions are utilized for additive manufacturing processes with laser metal deposition. As the name implies, the considered powders consist of metallic elements with varying powder particle sizes as one of the main characterization parameter. They are starting typically at approximately $45 \mu \mathrm{m}$ and go up to around $150 \mu \mathrm{m}$ or $200 \mu \mathrm{m}$. However, this means that the LMD process has higher grain sizes than the comparable AM process of selective laser melting (SLM). This among others is the reason for a better surface quality in SLM as higher median particle sizes increase surface roughness [10]. Lower grain sizes for LMD than the stated are in general not considered as flowability decreases [11] which leads also to a decrease in conveyability.

Another influential powder parameter is the density which can be described with the material density as well as bulk density (also called apparent density) and tapped density. The quotient between tapped density and bulk density is called the Hausner ratio and is an indicator for the flowability of the powder [12]. The flowability is also influenced by the shape of the respective particles and the moisture content of the powder and humidity levels [13]. The latter does not only affect the flowability, but also the manufacturing process itself, e.g., through increased oxidation [14].

The powder utilized in this publication is Nibasit 625-P, an Inconel alloy manufactured by the Deutsche Edelstahlwerke (DEW). It is a nickel-based powder 
with mass fractions in percent for chromium (22\%), molybdenum (9\%), iron (4\%), niobium (3.6\%) and parts of carbon $(<0.5 \%)$. The particle sizes ranged from 45 to around $150 \mu \mathrm{m}$. The particle size distribution with the resulting relative and cumulative frequency in $\%$ is given in Fig. 2a. It shows a median particle size of $d_{50}=$ $74.4 \mu \mathrm{m}$. The measurements were conducted with a multilaser particle size analyzer. Furthermore, the utilized powder is shown with a light microscope at $\times 200$ magnification in Fig. 2b.

The approximate material density of the powder was at $8.6 \mathrm{~g} / \mathrm{cm}^{3}$. The bulk density was determined at $4.6 \mathrm{~g} / \mathrm{cm}^{3}$ and the tapped density at $5.1 \mathrm{~g} / \mathrm{cm}^{3}$ which led to an Hausner ratio of around 1.11. This indicated a flowability directly between "excellent" and "good" with a "very free" to "free flow" [12]. The flowability was also checked visually and was assessed to be good without significant formation of clusters. Other parameters of the powder like detailed humidity levels or detailed particle shape specifications were not specifically considered here. Besides the fact that they are more difficult to measure and qualify, they might be taken into account for future works with variant types of powder for the presented in situ measurement and closedloop control. Furthermore, the usage of recycled powders which were part of an LMD process before, but did not end up being processed, might be investigated in this context.

\subsection{Tubes and flow path}

The tubes for transporting the powder from the feeder also have an influence on the conveying behavior with their routing, previous period of application, and length. The tubes utilized in this publication had an outer diameter of $6 \mathrm{~mm}$ and an inner diameter of $4 \mathrm{~mm}$. They were made from Polyurethan by Festo and were antistatic. This was necessary as the movement of the powder particles along the tube walls can cause static loadings to occur through the triboelectric effect, especially with the high electric resistance of the Polyurethan material. These loadings are unwanted as it might affect the powder flow itself or the LMD process. The charges are hard to control and complicate the realization of a steady process. The utilized tubes were not brand new, so that the conveyed powder did not adhere on the tube walls. This tendency can be observed during the first uses which can cause fluctuations in the actual powder mass flow rate. After a while, a thin layer of powder settled on the tube walls and the effect does not occur anymore. In the experiments, the tubes took the direct vertical path from the powder feeder to the glass beaker beneath to reduce possible influences with the decreased tube length of approximately $0.8 \mathrm{~m}$.

\subsection{Powder sensing}

For this publication, two ways of recording the powder flow over time were applied: a gravitational measurement with a weighing scale on the one hand and an in situ approach with an optical sensor on the other hand. With this setup, calibration and validation practices were performed.

For the direct powder mass flow rate measurement, the regarded powder was conveyed through the aforementioned tube into a glass beaker located on a weighing scale. It showed the increasing total amount of powder that was conveyed over the course of the experiments. The tube was hold in place with a stand without touching the beaker. A layer of foam closed the beaker opening to prevent powder from exiting. Before every measurement, it was waited until the resulting amount of carrier gas inside the beaker reached a steady state. This is important as the density of argon is higher than the density of air which can cause

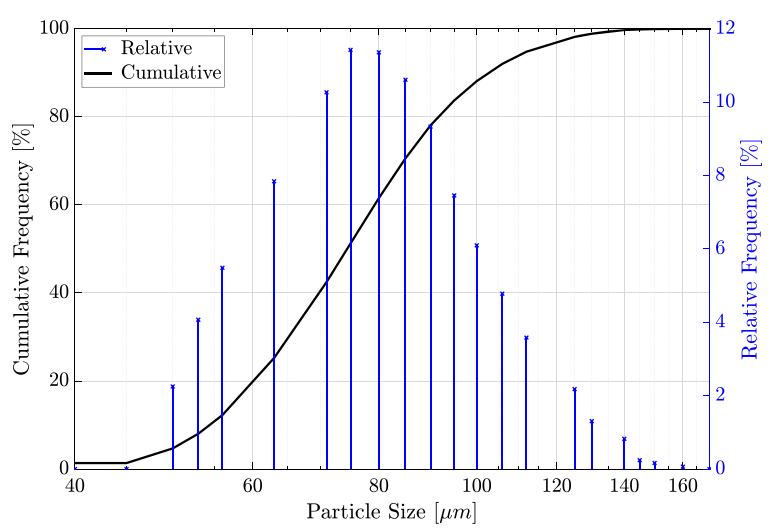

(a) Measured powder particle size distribution where the relative- and the cumulative frequency of the particle sizes is given in $\%$.

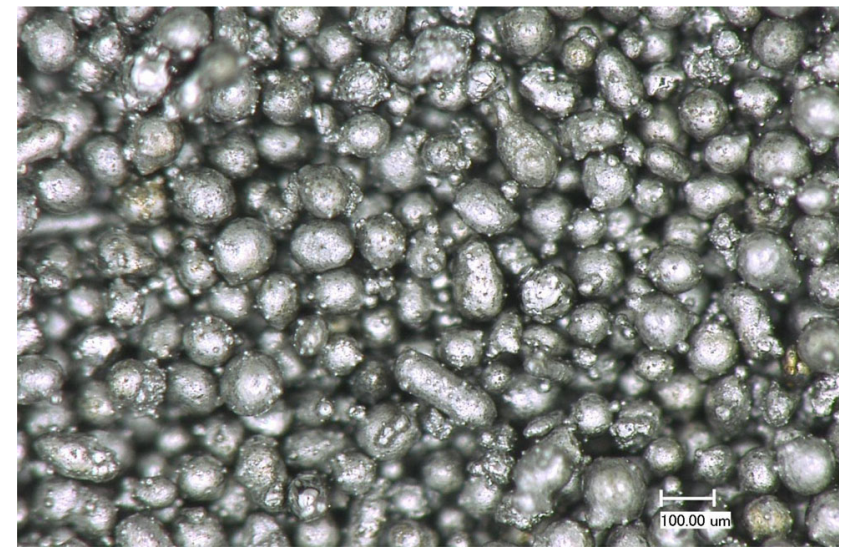

(b) Light microscopic image of the powder at 200x magnification.

Fig. 2 Extended information regarding the utilized Inconel alloy powder IN625-P 
inaccuracies in weight recordings. With these measures, unwanted disturbances were minimized, and a higher precision of the measurement was achieved. The weight information along with the timestamps were transmitted to a computer at approximately $3 \mathrm{~Hz}$ and saved into a file. This measurement principle directly gives the actual amount of powder conveyed over time but is obviously not applicable during an LMD process. The powder flow is interrupted and is not useable for further processing anymore.

The indirect in situ measurement was conducted with a simple and enclosed optical system by Medicoat called FlowWatch. An aspect supporting the sensor choice was its availability and the fact that some LMD powder supply systems have this identical sensor already installed for very basic monitoring purposes. This underlines its applicability and the low boundaries for implementing the proposed more sophisticated approach in an industry context. For the experiments, the sensor was located in the powder path right after the vibration feeder outlet. Nevertheless, other external influences and the effect of the sensor orientation were assessed qualitatively. External vibrations along with sudden small impacts did not have a notable influence on the recorded sensor signal for the prevailing flow conditions. This speaks for an applicability in industrial environments additionally to the enclosed and robust nature of the sensor. The same was observed when changing the orientation of the sensor (vertical and horizontal) as well as the position (directly at the feeder outlet and further downstream): each of the instances did not have any notable effects on the sensor output in relation to the conveyed powder flow. However, rapid movements due to an installation close to the LMD nozzle can not be ruled out at this point. They have to be determined in future works when focus lies on the manufacturing of components with the presented system and its further qualification for industrial applications.

The optical sensor itself consists of a glass tube through which the powder is transported without being disturbed. Radially to it, an infrared light signal at $850 \mathrm{~nm}$ is transmitted through the glass tube. A receiver on the other side of the tube detects the transmitted light signal which is dependent on the amount of moving powder in the glass tube. The higher the amount of powder and therefore the area blocking light on its way, the higher is the obscuration of the light signal. It has to be noted that the obscuring powder area is not directly dependent on the powder mass flow rate, but on the powder volume rate. With rising powder volume rates, the voltage sensor output rises as well. But as stated before, the sensor output and therefore the obscuration behavior is largely independent from the sensor's orientation and position in the flow path for typical flow conditions. The output signal is given with $0 \mathrm{~V} \mathrm{DC}$ for no powder flow and is limited to a maximum of $10 \mathrm{~V} \mathrm{DC}$.
It underlines that this method is solely an indirect way of powder flow measurement. No mass flow rate is sensed, but a volumetric amount given as voltage output.

\subsection{Setup and data collection}

The center point of the experiments was a numerical control (NC). It controlled the work stroke and the carrier gas flow rate of the vibration feeder. The optical powder sensor was connected to the $\mathrm{NC}$ for both, power supply and data recording. The utilized NC was a Siemens Sinumerik 840D. The vibration feeder was connected via Profinet while the sensor used an analog connection. With the NC, the experimental procedures along with the LMD process itself were programmed in a G-code with functionalities like synchronized actions. These are looped commands which are executed constantly or when a monitored condition is given. The frequency for condition checks and command execution was at $8 \mathrm{~ms}$. This resulted in a control loop frequency and data recording rate of $125 \mathrm{~Hz}$.

The Sinumerik recorded the data from the vibration feeder and the optical sensor over the course of the experiments. This information was saved for every time step with the trace functionality of the Sinumerik and was written into a file. The methods for creating the wanted data collections with this setup are shown in the following section.

\section{Methodology}

In the following, the methods and procedures are described to accomplish the two main aspects of this publication: the implementation of an in situ powder mass flow rate measurement with the optical sensor as well as a closedloop powder mass flow rate control based on said sensor signal.

\subsection{Sensor measurement}

As described before, the powder mass information $m$ in $\mathrm{g}$ with the corresponding timestamps was recorded in a file. To receive the mass flow rate $\dot{m}$ in $\mathrm{g} / \mathrm{min}$, the difference quotient was calculated. A simple moving average filter with a window size of $1.5 \mathrm{~s}$ was used to smoothen the result and to remove outlier measurements for plotting purposes. To receive the correct relation between optical sensor output and actual powder mass flow rate, a valid data fusion was carried out. This was done by mapping the corresponding timestamps of the powder mass flow rate from the difference quotient to the trace file timestamps. As the weight information was recorded at a lower frequency, its data points were interpolated to match the Sinumerik 
trace. Between the data from the Sinumerik and from the weighing scale, a time delay was still present at this point. As the signals from recorded mass flow rate and sensor output correspond to each other over time, a crosscorrelation between the two was carried out. The time delay between the two signals was at the point in time where the correlation value reached its maximum. With this, the powder mass flow rate information was shifted in time accordingly. This also eliminated any delays occurring due to the distance between the optical sensor directly at the vibration feeder and the weighing scale. Therefore, the time the powder needed to travel through the tube did not have an influence and both data collection instances were synchronized. Correlation values of over 0.97 between the measured powder mass flow rate and the sensor output also show that the optical sensor is suitable for an indirect powder mass flow rate evaluation.

To determine the dependency between the synchronized optical sensor output and the actual powder mass flow rate, the vibration feeder was run at changing feeder speeds with a constant carrier gas flow rate. The controlling was done by the NC. The resulting powder flow was recorded via the optical sensor in $\mathrm{mV}$ as well as with the calculated powder mass flow rate from the weighing scale in $\mathrm{g} / \mathrm{min}$. This procedure was repeated for three different argon carrier gas flow rates: $4.5 \mathrm{l} / \mathrm{min}, 6 \mathrm{l} / \mathrm{min}$, and $9 \mathrm{l} / \mathrm{min}$. With this, most of the range of typical carrier gas flow rates was covered.

The next step was to evaluate the dependence of the powder mass flow rate and the carrier gas flow rate on the optical sensor output. The idea was to eliminate any dependencies of time and feeder speed. This was done by averaging the recorded powder mass flow rate and the sensor output over a set time window. A time window of five seconds produced satisfying results in terms of data point amount and data point dispersion. These data points held the relation between sensor value and powder mass flow rate. Power functions were used to describe the mathematical dependency between the two. The format shown in Eq. 1 was utilized where $S$ is the sensor value, $\dot{m}$ the powder mass flow rate, and $a$ and $b$ are the free parameters of the function. For the fitting of these two parameters, a nonlinear least squares approach was chosen with a trust region algorithm [15].

$S(\dot{m})=a \cdot \dot{m}^{b}$

To validate the thereby found relation between powder mass flow rate and sensor output, a completely separate data recording was conducted for a carrier gas flow rate of $\dot{V}=6 \mathrm{l} / \mathrm{min}$. The previously found function was rearranged to the form in Eq. 2.

$\dot{m}(S)=\sqrt[b]{\frac{S}{a}}$
The recorded optical sensor output was recalculated with said equation to receive the corresponding mass flow rate. It was plotted along with the powder mass flow rate recorded by the weighing scale. The latter was smoothed by a moving average filter with a window size of $1.2 \mathrm{~s}$ in this case for better comparability. The two obtained graphs were compared visually to evaluate the validity of the optical sensor for in situ mass flow rate measurements. This showed the behavior and the level of fitting for the two sensor signals.

\subsection{Powder control}

The introduced method of sensing the powder mass flow rate during the process was utilized for a feedback path back to the vibration powder feeder. With the feeder as an actuator, a closed-loop control was implemented. A singleinput single-output (SISO) system is present with the feeder speed as input and the sensor signal as output parameter.

To investigate this system, step response experiments were conducted. Prior tests suggested the assumption that the step response behavior depends on the feeder speed level ran before the examined step. To quantify this effect and to ensure repeatability, a constant feeder speed was active before the step for a time of around two to three minutes until a steady state was reached. Afterwards, the feeder was stopped, and the step response was performed. Both the feeder speed as input and the sensor signal as output were recorded. Three different operating points with corresponding feeder speeds were determined: "low" (42\%), "medium" (64\%), and "high" (83\%). With this, a linearization of the non-linearity between feeder speed and sensor output took place. The speeds match the powder mass flow rates of approximately $\dot{m}=3.1 \mathrm{~g} / \mathrm{min}, \dot{m}=$ $11.1 \mathrm{~g} / \mathrm{min}$, and $\dot{m}=21.7 \mathrm{~g} / \mathrm{min}$ respectively when a steady state is reached. In this way, typical but different levels of powder mass flow rates were examined for the system's dynamic behavior. The mentioned feeder speeds were not only adapted for the height of the step response, but also for the steady state before the step. So for example, the "low" step response to $42 \%$ feeder speed was investigated three times: once with the same feeder speed of $42 \%$ beforehand and twice with a different feeder speed before-in this case $64 \%$ and $83 \%$. With this, the influence of the preceding feeder speed on the step response outcome was determined with a total of nine different configurations. Furthermore, they were tested for the three carrier gas flow rates of $4.5 \mathrm{l} / \mathrm{min}, 6 \mathrm{l} / \mathrm{min}$, and $9 \mathrm{l} / \mathrm{min}$ which results in a total number of 27 configurations.

The found information from the step experiments was utilized to implement a closed-loop control cycle. The Sinumerik and G-code was used as similarly done by [16]. A PID controller approach was applied as it is wide-spread, 
so numerous practices for tuning the controller gains are available. The individual PID controller statements were transferred into G-code line by line [17]. Three controller gains had to be approximated: $K_{P}$ (proportional part), $K_{I}$ (integral part), and $K_{D}$ (derivative part). They were calculated from the step responses described before. For the calculation, the "T-Sum" approach was chosen as it is a direct way to evaluate appropriate gains with a robust behavior. Compared to other classic PID tuning rules like Ziegler-Nichols, the obtained controller behavior is less aggressive while preventing overshooting [18]. This made it very suitable for the use case as abrupt changes were not expected and the increased powder mass flow rate of overshoots was avoided. The two important parameters for the T-Sum method are the stationary gain $K_{S}$ and the sum time constant $T_{\Sigma}$. The stationary gain $K_{S}$ is the gain factor between input and output of the system. In this case, between the powder feeder amplitude in $\%$ and the sensor output in $\mathrm{mV}$. In the presented cases, the feeder amplitude was therefore $42 \%$ (low), 64\% (medium), and $83 \%$ (high) while the sensor output was the final value reached after the step without overshoot. The sum time constant $T_{\Sigma}$ in $\mathrm{s}$ as the other important parameter is a measure of how fast the output reacts and adapts to a change in the input. It is a point in time during the step and lies between the step initiation and the time at which the peak value is reached. $T_{\Sigma}$ was calculated from the data gathered in the step response experiments. To do so, two areas at the graph were considered: the area under the graph after the step initiation up until $T_{\Sigma}$ and the area after $T_{\Sigma}$ over the graph limited by the peak value. $T_{\Sigma}$ is the point in time at which the two mentioned areas have the same size. With a selfwritten script, the respective sum time constant $T_{\Sigma}$ was found from the data at which an equilibrium of the two areas was reached. The relations between $K_{S}$ and $T_{\Sigma}$ to the wanted controller gains $K_{P}, K_{I}$, and $K_{D}$ are shown in the Eqs. 3, 4, and 5.

$$
\begin{aligned}
K_{P} & =\frac{1}{K_{S}} \\
K_{I} & =\frac{K_{P}}{0.66 \cdot T_{\Sigma}} \\
K_{D} & =0.167 \cdot K_{P} \cdot T_{\Sigma}
\end{aligned}
$$

With the parameters $K_{S}$ and $T_{\Sigma}$ gathered from the data recordings, the 27 controller gain triplets $K_{P}, K_{I}$, and $K_{D}$ were calculated and compared. To further improve the controller performance, a saturation was implemented so that the powder feeder could not enter extreme feeder speeds. This is especially important during large changes in the nominal value like for the investigated step responses. The saturation was set at $\pm 15 \%$ feeder speed (absolute) around the initial feeder speed. Another means for an improved controller performance is the introduction of antiwindup. When abrupt changes are taking place and the actual sensor value is far off the nominal value, the integral part of the controller logic can accumulate enormous values in a fast manner. Various ways of anti-windup and therefore I-Part limitation are possible. In this publication, the I-Part of the controller was set to zero whenever the actual sensor value was not close enough to the nominal value. This prevented from an unwanted rise of the I-Part which makes the integral control inaccurate and can cause disturbances. The integral control region in which the I-Part was not set to zero was at $\pm 20 \%$ from the nominal sensor value. So when the actual sensor value was within this region, the integral control part of the PID controller was active. The more drastic deviations outside this region were compensated with the P- and the D-Part as this is their main field of application.

To finally evaluate the PID controller during its usage, nine controller gain sets of the 27 total configurations were tested. They were chosen in the way that for every of the three "step" and "before step" levels, every gas flow rate was utilized exactly once. By this, a maximized variety of controller gains was tested. A step response was performed with the controller and its respective gains enabled. This included the aforementioned saturation and anti-windup functionalities. The obtained data was recorded and compared to the data without active controller to examine the improvements in terms of dynamic behavior and accuracy to the nominal value. To do so, the mean relative error $(M R E)$ [19] was utilized. Its formula for this application is presented in Eq. 6 .

$\operatorname{MRE}[\%]=\frac{\sum\left|S_{\text {nom }}-S_{\text {act }}\right|}{S_{\text {nom }} \cdot n_{\text {meas }}} \cdot 100 \%$

The mean relative error calculates the sum of the absolute differences between the nominal sensor value $S_{\text {nom }}$ and the actual sensor value $S_{a c t}$ during the running process for every time step. It is normalized with the nominal sensor value multiplied by the number of measurement time steps $n_{\text {meas }}$. A multiplication by 100 results in a percentage. The MRE gives information about the recorded sensor output deviation from the nominal sensor value. Due to the normalization with the nominal sensor value, a comparison between the different carrier gas flow rates was possible as their level differ for the same powder mass flow rate.

\section{Results and discussion}

The previously introduced methodology for the in situ powder mass flow rate measurement via optical sensor and the closed-loop powder control was applied. The obtained results are presented and discussed in the following. 


\subsection{Sensor measurement}

The results for the simultaneous measurements with optical sensor and weighing scale at varying feeder speeds are shown in Fig. 3. The optical sensor output is plotted in $\mathrm{mV}$ at the three different argon carrier gas flow rates $4.5 \mathrm{l} / \mathrm{min}$ (green), 6 1/min (red), and 9 1/min (black). The feeder speeds in $\%$ are shown in blue as well as one exemplary mass flow rate recorded with the weighing scale in $\mathrm{g} / \mathrm{min}$ at $9 \mathrm{l} / \mathrm{min}$ (black dotted). The mass flow rate results for the other two gas flow rates are not shown as they were naturally at practically identical rates. Reason is that the carrier gas flow rate does not interfere with the amount of powder that is conveyed.

The graphs show the direct dependence of the optical sensor output and the actual powder mass flow rate in a very good manner. The fluctuations over time of the recorded powder mass flow rates are also clearly visible. An overshooting behavior can be seen clearly in Fig. 3, especially for rising steps in feeder speed above $60 \%$ at around 120 to $220 \mathrm{~s}$. On the other hand, Fig. 3 shows also an undershooting behavior for decreasing feeder speed steps between 55 and $75 \%$ at around 270 to $360 \mathrm{~s}$.

Even at a constant feeder speed, changing mass flow rates were present. But the general sensor value trends were rather similar for all three gas flow configurations. That shows that a general repeatability with this specific powder at the identical feeder speed sequence was given. As noted before, the powder mass flow rate values were at the same level for all three carrier gas configurations. In contrast, the optical sensor outputs showed the same behavior over time, but at changing output levels. With increasing gas flow rates, the sensor output level decreased. This inverse proportionality can be explained with the measurement

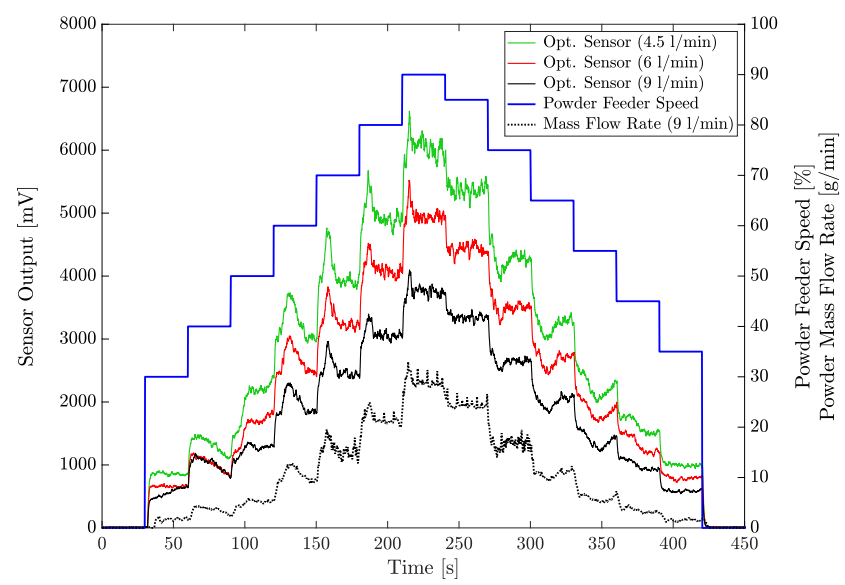

Fig. 3 Powder conveying for varying vibration feeder speeds at $4.5 \mathrm{l} / \mathrm{min}, 6 \mathrm{l} / \mathrm{min}$, and $9 \mathrm{l} / \mathrm{min}$ carrier gas flow rate. The resulting optical sensor outputs and an exemplary powder mass flow rate over time along with the feeder speeds are presented principle of the optical sensor. It measures the obscuration of the infrared light due to the powder particles traveling through the sensor and therefore through said infrared light (see Section 2.4). As the carrier gas volume flow rate $\dot{V}$ rises, the gas as well as the powder particles being conveyed by it has a higher velocity. For the same amount of powder, but conveyed with more carrier gas, the particles move faster through the sensor. This means that the particles are located within the sensing infrared light for a shorter period of time, more light reaches the opposing receiver, and the obscuration is lower. This results in a lower voltage sensor output.

It is also apparent that feeder speed and optical sensor value do not have a linear dependence. For example, at 4.5 1/min (green in Fig. 3) the sensor output was around $1000 \mathrm{mV}$ with a feeder speed of $30 \%$. With a feeder speed of $60 \%$, the sensor output was at around $3000 \mathrm{mV}$ after the overshoot. So with double the feeder speed, the sensor output was three times higher while at $0 \%$ the output is $0 \mathrm{mV}$. Therefore, a non-linear dependency is present.

Based on these measurements, the mathematical dependency between optical sensor output and powder mass flow rate was evaluated with the described averaging and fitting methods. The results are illustrated in Fig. 4.

It shows a very good match between the fitted curves and the respective datapoints. Due to this, the relation between in situ measured optical sensor output and actual powder mass flow rate follows the introduced power function. It revealed that the exponent can be set to $b=0.63$ for all three carrier gas configurations. Therefore, $b$ is independent from the carrier gas flow rate while the same powder and the

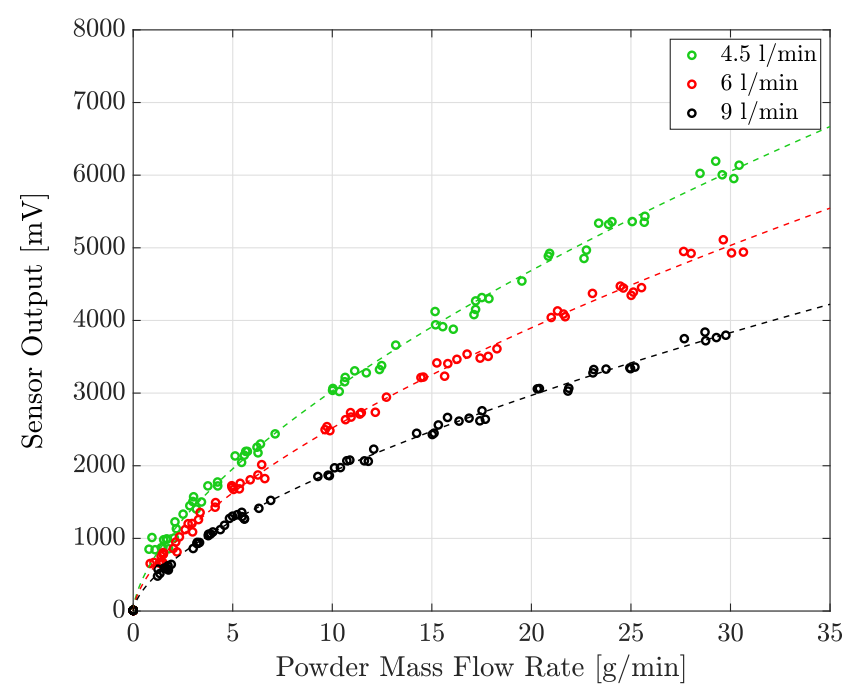

Fig. 4 Dependency of averaged sensor output $(\mathrm{mV})$ on the averaged mass flow rate $\dot{m}(\mathrm{~g} / \mathrm{min})$ for the three tested carrier gas flow rates. The averaging time window for the data points was five seconds. Furthermore, the fitted power function for each of the three cases is plotted 
Table 1 Scaling factors $a$ and exponent $b$ of the function $S=a \cdot \dot{m}^{b}$ for the three carrier gas flow configurations

\begin{tabular}{lll}
\hline Carrier gas $\dot{V}$ & $a$ & $b$ \\
\hline $4.5 \mathrm{l} / \mathrm{min}$ & 710.1 & 0.63 \\
$6 \mathrm{l} / \mathrm{min}$ & 590.5 & 0.63 \\
$9 \mathrm{l} / \mathrm{min}$ & 449.6 & 0.63 \\
\hline
\end{tabular}

same powder feeder line is considered. The results for the three scaling factors $a$ are shown alongside $b$ in Table 1 . The physical unit of the factor $a$ is given with $(\mathrm{mV} \mathrm{min} / \mathrm{g})^{0.63}$ for completeness, but is not further mentioned in the following.

It shows that with increasing carrier gas flow rate, the factor $a$ is decreasing. This is rooted in the aforementioned inproportional behavior between optical sensor output and gas flow rate. With more carrier gas, the output is lower as powder concentration is lower even though the mass flow rate is identical.

With the shown parameters, the conversion between optical sensor output in $\mathrm{mV}$ and actual powder mass flow rate in $\mathrm{g} / \mathrm{min}$ is possible. To validate this, the completely separate measurement row of optical sensor and weighing scale is shown in Fig. 5. The sensor output in it is converted with Eq. 2 into a powder mass flow rate.

It shows that the direct and the indirect measurement are both aligning very well while even distinctive peaks are presented correctly with the optical sensor measurement. The course of the directly measured powder mass flow rate underlines again the need of the previously applied

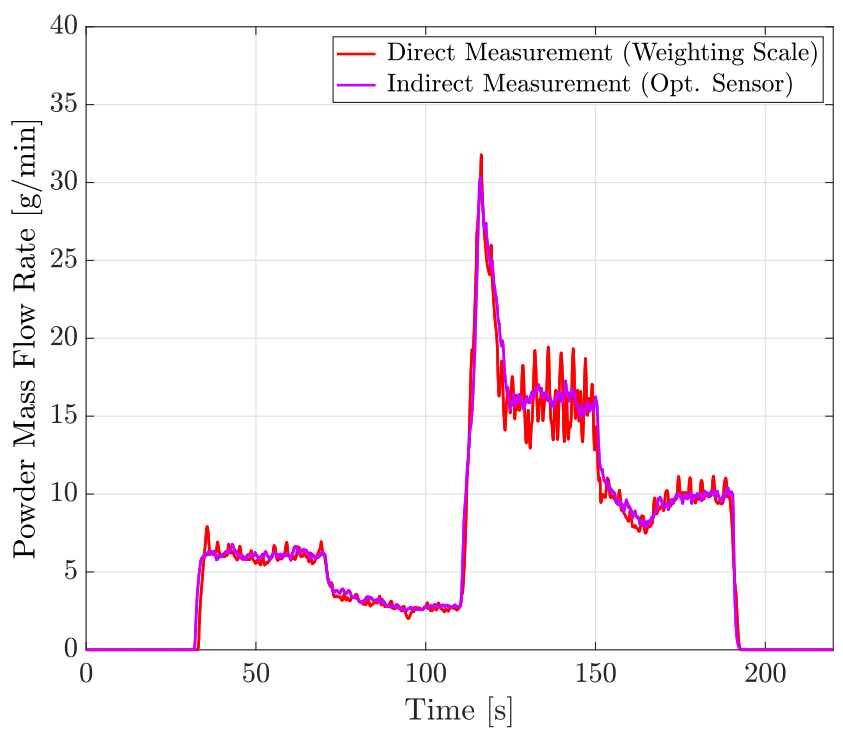

Fig. 5 Validation of the previously found dependency $S=a \cdot \dot{m}^{b}$ between actual powder mass flow rate $\dot{m}$ and the sensor output $S$. The indirectly measured powder mass flow rate was calculated from the sensor output to obtain a powder mass flow rate. A completely separate measurement run at the carrier gas flow rate $\dot{V}=61 / \mathrm{min}$ is shown averaging method in Fig. 4. Fluctuation and especially oscillations are very present at some points which are not likely to occur in this strong manner. Reason for this might be the rather low measurement frequency of the weighing scale at approximately $3 \mathrm{~Hz}$. Furthermore, the signal of the sensor is more stable and reasonable while fluctuations and peaks are depicted well. The recording frequency is much higher in comparison at $125 \mathrm{~Hz}$. With this, it suggests that the optical sensor measurement technique might be more reliable and less vulnerable to disturbances than the direct measurement via weighing scale. Nevertheless, a weighing scale measurement is still needed to find the correct relation between optical sensor output and actual powder mass flow rate. When this is found, the optical sensor represents a simple and reliable way of in situ powder mass flow rate measurement during an LMD process which was not available before. It does not affect the flow of the powder in any way as it is an integrated part of the flow path. The installation is straightforward as it only has to be connected to the powder tubing. Therefore, retrofitting is no issue in any way.

\subsection{Powder control}

With the in situ measurement, a closed-loop control for the powder mass flow rate was implemented. For this, the dynamic behavior of the system was investigated. As shown before, a non-linear dependency between feeder speed (input) and sensor value (output) is present. Overshooting and undershooting was detected in different forms for stepwise changes in feeder speed. The step responses at the three operating points "low" (42\%), "medium" (64\%), and "high" (83\%) dependent on the active feeder speed level before the step were performed and recorded as described in Section 3.2. Its results are shown in Fig. 6 for the same carrier gas flow rates also considered before.

The general courses at the same configuration are independent from the amount of argon carrier gas flow as it only changes the amplification likewise to the observations before. But Fig. 6 clearly shows the influence on the response of the feeder speed level and therefore the influence of the amount of powder transported before the step. The relative values of the peaks and lows as well as their duration are differing for one carrier gas setting. This proves that the previous powder mass flow rates have an influence on the current mass flow rate. This is evidence that the system inheres a memory effect.

The behavior can be separated into three categories. For the first, the output signal was not over- or undershooting when the same feeder speed level was running before and after the step. This marks the three plots on the top left to bottom right diagonal. The three plots below this diagonal show a distinctive peak from which the signal went into a 

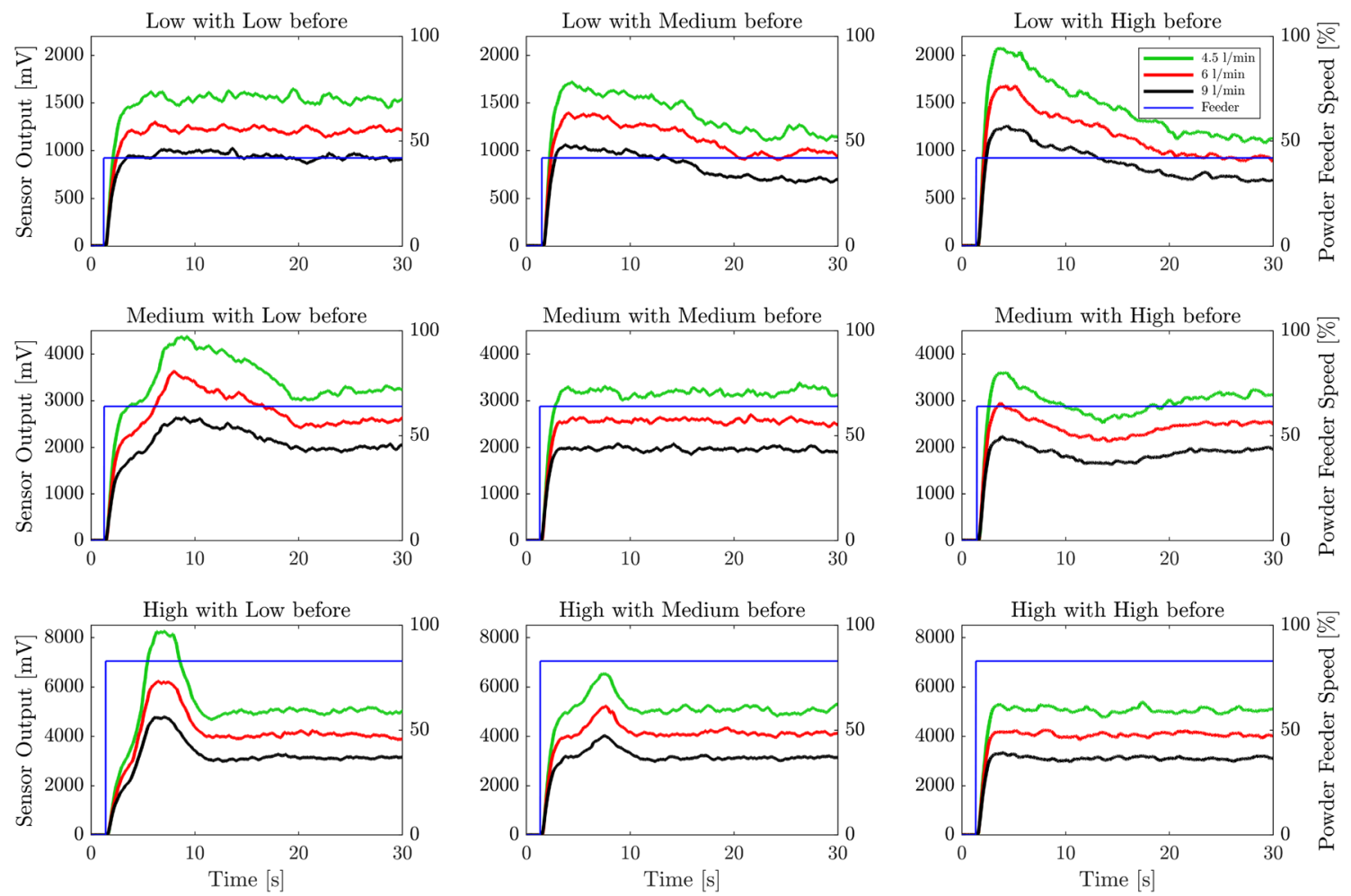

Fig. 6 Optical sensor values for the powder flow over time with a vibration feeder speed step response. The height of the step is varied as well as the feeder speed before the step execution. The three feeder speed levels are 42\% ("low"), 64\% (“medium"), and 83\% ("high"). Furthermore, the three investigated carrier gas flow rates are presented constant value after some seconds. The peak was reached with a gradient which declines temporarily on the way to the peak. The height of the peak was set by the difference of the feeder speeds. The higher the difference, the higher was the peak. The width of the peak was influenced by the speed after the step. With a lower speed, the peak was wider, so it took more time until a constant level was reached. This all marks a second category in which a lower feeder speed was active before the step. The last category are the three plots above the aforementioned diagonal which had a bigger feeder speed before the step than after it. They had a peak as well, but it is not as distinctive, and it was reached with a constantly high gradient. From there, the signal slowly decreased. With a higher powder feeder speed ("medium"), the output signal rose after some time to a constant level. The two "low" feeder speed settings did not reach the same level as the most-left step response on the diagonal as their signal apparently just started to rise at the end of the examined time window. But in general, the other seven step responses reached approximately the same level with the same feeder speed after the step.

This dynamic behavior can be described with the design of vibration feeders. The variations are rooted in the transport of the powder along the vibrating metal chute and its amount on it. When the feeder is started at a certain feeder speed level and the same feeder speed was active beforehand, the conveying process is continued without any major fluctuations and no overshooting is occurring. If this is not the case and a different feeder speed was active before the feeder speed start, a surge of powder is leaving the feeder and an overshooting is present. This underlines again the need of a controlled powder conveying process.

Based on the presented step response data recordings, the controller gains $K_{P}, K_{I}$, and $K_{D}$ were calculated with the T-Sum method. The results are shown in Tables 2, 3 and 4. Note that the displayed values are multiplied by $10^{2}$ for a better overview and comparability. The location for the step configurations in the three by three grid of the tables corresponds to the same locations as in Fig. 6.

The calculated controller gains are all situated roughly at the same order of magnitude. It shows that the gains were increasing for increasing carrier gas flow rates. That was the case for all three gains $K_{P}, K_{I}$, and $K_{D}$. Reason is that the general level of the sensor output is lower with higher gas flow rates as shown in Section 4.1. With this and the inverse proportionality in Eq. 3, the value for $K_{P}$ was increasing with increasing gas flow rate. Following this, the values for $K_{I}$ and $K_{D}$ behaved in the same manner as Eqs. 4 and 5 show the direct dependency from $K_{P}$. Furthermore, $K_{P}$ was virtually the same for every row 
Table $2 K_{P} \cdot 10^{2}$ for the three investigated carrier gas flow rates

\begin{tabular}{llll}
\hline Step & \multicolumn{3}{c}{ Before step } \\
\cline { 2 - 4 } & Low & Med. & High \\
\hline (a) Carrier gas $\dot{V}=4.51 / \mathrm{min}$ & 2.7 & 2.7 \\
Low & 2.7 & 2.04 & 2.03 \\
Med. & 2.04 & 1.62 & 1.67 \\
High & 1.69 & & \\
(b) Carrier gas $\dot{V}=61 / \mathrm{min}$ & & 3.41 \\
Low & 3.41 & 3.41 & 2.5 \\
Med. & 2.53 & 2.52 & 2.06 \\
High & 2.07 & 2.03 & \\
(c) Carrier gas $\dot{V}=91 / \mathrm{min}$ & & 4.51 \\
Low & 4.51 & 4.51 & 3.25 \\
Med. & 3.22 & 3.31 & 2.69 \\
High & 2.65 & 2.67 & \\
\hline
\end{tabular}

entry at the same gas flow rate. This was the case as the steady gain $K_{S}$ is nearly identical among the three entries in a row. They shared the same feeder speed and the same steady state value of the optical sensor. The latter was only slightly changing due to small variations in the step experiments. As $K_{P}$ was only dependent on $K_{S}$, the values were nearly identical. The two $K_{P}$ values for the "low" step with "medium" and "high" feeder speeds before were copied from the case where "low" was active before. This was due to the circumstance that a steady state was not yet reached at the end of the recording period. This marks the logical procedure according to the properties of $K_{P}$ presented before. Another interesting aspect is the course of

Table $3 K_{I} \cdot 10^{2}$ for the three investigated carrier gas flow rates

\begin{tabular}{llll}
\hline Step & \multicolumn{3}{c}{ Before step } \\
\cline { 2 - 3 } & Low & Med. & High \\
\hline
\end{tabular}

(a) Carrier gas $\dot{V}=4.51 / \mathrm{min}$

$\begin{array}{llll}\text { Low } & 4.2 & 4.74 & 4.97 \\ \text { Med. } & 1.32 & 3.79 & 3.76 \\ \text { High } & 1.0 & 1.4 & 3.32\end{array}$

(b) Carrier gas $\dot{V}=6 \mathrm{l} / \mathrm{min}$

$\begin{array}{llll}\text { Low } & 5.44 & 6.11 & 6.43 \\ \text { Med. } & 1.59 & 4.76 & 4.69 \\ \text { High } & 1.32 & 1.72 & 4.25\end{array}$

(c) Carrier gas $\dot{V}=91 / \mathrm{min}$

\begin{tabular}{llll} 
Low & 8.2 & 8.64 & 8.49 \\
Med. & 2.18 & 7.0 & 6.31 \\
High & 1.73 & 2.35 & 5.89 \\
\hline
\end{tabular}

Table $4 K_{D} \cdot 10^{2}$ for the three investigated carrier gas flow rates

\begin{tabular}{llll}
\hline Step & \multicolumn{3}{c}{ Before Step } \\
\cline { 2 - 4 } & Low & Med. & High \\
\hline (a) Carrier Gas $\dot{V}=4.51 / \mathrm{min}$ & & 0.37 \\
Low & 0.43 & 0.38 & 0.27 \\
Med. & 0.79 & 0.27 & 0.21 \\
High & 0.71 & 0.47 & \\
(b) Carrier Gas $\dot{V}=61 / \mathrm{min}$ & & 0.45 \\
Low & 0.53 & 0.48 & 0.33 \\
Med. & 1.0 & 0.33 & 0.25 \\
High & 0.81 & 0.6 & \\
(c) Carrier Gas $\dot{V}=91 / \mathrm{min}$ & & 0.6 \\
Low & 0.62 & 0.59 & 0.42 \\
Med. & 1.19 & 0.39 & 0.31 \\
High & 1.02 & 0.76 & \\
\hline
\end{tabular}

the respective $K_{P}$ and $K_{I}$ values for the same carrier gas flow rates. As the feeder speed of the actual step ("step") increases, the gain values are decreasing. The gain $K_{D}$ is not showing this behavior in any coherent way. The variety of different specific controller gains underlines the difficulty of selecting the optimal parameters. So for every dynamic requirement, the specific gain parameter should be chosen at this point.

The validation of the found controller gains was performed for nine of the 27 possible configurations according to Section 3.2. The results for the uncontrolled (brown) and the controlled (blue) case are shown over time in Fig. 7. Additionally, the nominal values (dashed) are plotted for better comparison.

The graphs show the improved performance of the controlled powder flow compared to the uncontrolled cases. The carrier gas flow level did not seem to have an influence on the controller performance and was therefore independent from it. The nominal values were in general reached faster with than without control. Furthermore, the nominal values were satisfied well with only minor deviations in the long run while responding faster. An improvement in dynamic and steady behavior was observed for all cases, but it was the smallest for the ones on the top left to bottom right diagonal. These were the cases in which the feeder speed before the step and the following feeder speed during the step were identical. They were even without control at a constant level throughout the course of the run without a significant overshoot occurring. Nevertheless, the controller helped to minimize steady state deviations from the nominal value.

The cases in which the feeder speed was higher before the step than the actual feeder speed for the step show a clear difference between controlled and uncontrolled instances. 

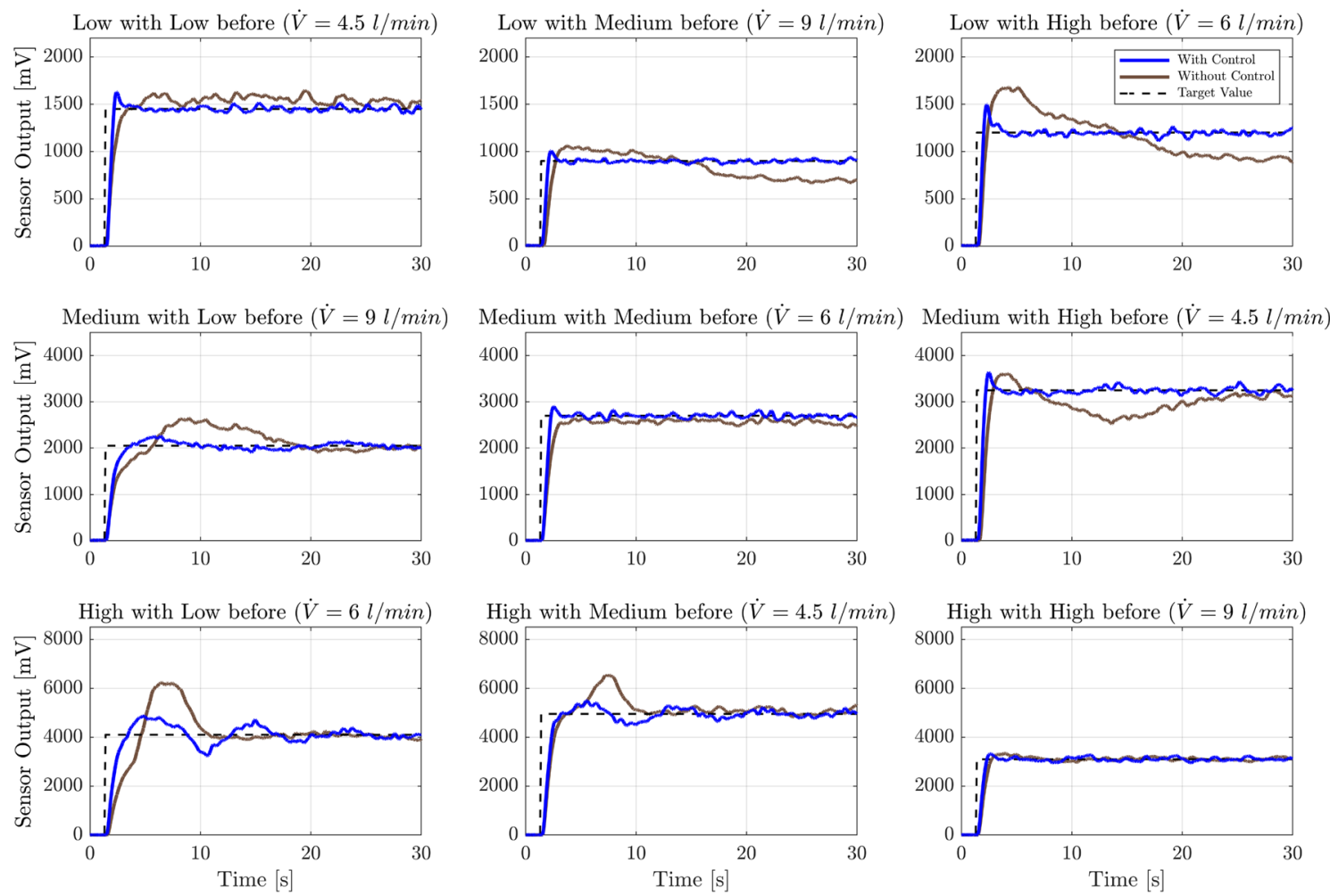

Fig. 7 Optical sensor values for the powder flow over time with a feeder speed step response. The uncontrolled (brown) and the controlled cases (blue) are presented as well as the nominal value (dotted)

They mark the three graphs in the top right corner above the aforementioned diagonal. The improvement with the PID controller was significant as the uncontrolled cases did not show a constant behavior. Instead, the sensor signal was slowly drifting. Therefore, distinctive differences to the nominal value were observable. Accordingly, the three graphs in the bottom left corner below the diagonal show also clear deviations from the nominal value in the uncontrolled case. Interestingly enough, the controller did not perform as good as for the other six investigated configurations. It still marked an improvement over the uncontrolled case as the sensor output rose faster in the beginning and the nominal value was reached. However, a more distinctive overshoot as well as slight oscillations around the nominal value was observed. Therefore, the tuning of the three controller gains for the cases where a lower feeder speed was active before the actual feeder speed step was not as optimized as for the other cases.

To finally confirm the observations regarding the controller performance qualitatively, the introduced mean relative error $(M R E)$ was calculated for the nine configurations. The results are presented in Table 5.

The observations from Fig. 7 are validated by the mean relative errors in Table 5. The lowest error values among the cases without control were on the aforementioned diagonal. With the PID controller active, the errors were further minimized. But the improvement was not as strong as it was for the three configurations in the top right corner. These were the instances in which the feeder speed before the step was higher than the feeder speed for the actual step response. A clear decrease in the $M R E$ was observed for them.

Accordingly, the deviations from the nominal value were also lower with the PID controller for the configurations in the bottom left corner. These were the cases where the feeder speed before the step was lower than the feeder speed for the actual step response. But matching to the statements

Table 5 Mean relative error [\%] without and with closed-loop control for the nine exemplarily tested step and carrier gas configurations

\begin{tabular}{|c|c|c|c|c|c|c|}
\hline \multirow[t]{3}{*}{ Step } & \multicolumn{6}{|c|}{ Before step } \\
\hline & \multicolumn{2}{|l|}{ Low } & \multicolumn{2}{|l|}{ Medium } & \multicolumn{2}{|l|}{ High } \\
\hline & Without & With & Without & With & Without & With \\
\hline Low & $8.9 \%$ & $3.3 \%$ & $16.4 \%$ & $3.2 \%$ & $19.7 \%$ & $3.6 \%$ \\
\hline Medium & $12.7 \%$ & $5.1 \%$ & $7.2 \%$ & $3.3 \%$ & $10.9 \%$ & $3.5 \%$ \\
\hline High & $12.9 \%$ & $8.5 \%$ & $7.9 \%$ & $5.5 \%$ & $3.9 \%$ & $3.6 \%$ \\
\hline
\end{tabular}


gathered from Fig. 7, their mean relative errors were the highest among the controlled cases. Improvements to the controller gains for preventing overshoot and oscillation are imaginable.

This means that the $M R E$ can be kept in any case below $9 \%$ and for the most step response configurations even below $4 \%$ with active closed-loop control. The improvement of the $M R E$ compared to the uncontrolled case during the step was at least over $7 \%$ (from 3.9 to $3.6 \%$ for "high before high") and was as high as over $81 \%(19.7$ to $3.6 \%$ for "high before low"). A clear optimization is therefore present.

\section{Summary and outlook}

This publication investigated the powder mass flow rate in the context of the AM process LMD as it is one of main parameters for the manufacturing process itself. Based on the presented problem statement, an in situ measurement concept along with a closed-loop control for the utilized vibration feeder was presented. Following main aspects were shown:

- The powder mass flow rate $\dot{m}$ is described with the output $S$ from the utilized optical sensor by $S=a \cdot \dot{m}^{b}$. With a higher argon carrier gas flow rate, the sensor value is lower at the same powder mass flow rate and therefore the factor $a$ is lower as well. However, the exponent is constant at $b=0.63$ for the investigated cases.

- Based on this, proof of concept of a retrofittable optical sensor system for in situ powder mass flow rate measurement at $125 \mathrm{~Hz}$.

- Finding of a memory effect for the vibration feeder system as the previous powder flow has a direct impact on the current powder flow. Reason is the changing amount of powder on the vibrating chute.

- The memory effect results in an overshooting followed by an undershooting over a broad time period for a step response when a higher feeder speed was active before. When a lower feeder speed was active before, a changing gradient leads to an overshooting before the steady state is reached.

- The duration of the memory effect influence depends on the current vibration feeder speed. With a higher feeder speed, the effect wears off faster and a steady state is reached in a shorter time.

- A closed-loop powder mass flow rate control is implemented successfully with a PID controller approach and the optical sensor signal. The vibration feeder itself with its feeder speed serves as actuator and the T-Sum method can be utilized to find appropriate controller gains.

- With the introduced closed-loop control, the mean relative error of the powder mass flow rate to its nominal value is kept below $9 \%$ for the presented step response configurations. For most of the configurations, it is kept even below $4 \%$.

- The reduction of the mean relative error with active controller over the uncontrolled case is at least $7 \%$ and can reach over $81 \%$ for the presented cases.

The introduced approach for powder mass flow rate sensing and control represents a novel and retrofittable method with high potential. Based on the demonstrated steps and the proof of concept, an implementation on other powder feeding systems is possible. While doing so, a limitation solely to vibration feeders is technically not given at this point, and a usage with suitable sensor functions and controller gains is probably feasible. The utilization with LMD promises a direct overall improvement of the manufacturing process due to increased repeatability and an introduction of a monitoring functionality. The latter is crucial for establishing AM methods in industries like aerospace as verifiability plays a key role. A range of available powder mass flow rates is presented which can be used to implement different $\dot{m}$ values, even in a single LMD build-up. As the previous powder mass flow rate is known, the controller gains for the next process step can be adjusted accordingly. In this way, a controlled powder flow is present throughout different LMD process phases with varying target values. If more operating points are needed, the corresponding controller gains can be easily determined with the shown methods. Furthermore, the apparent insensitivity against external influences marks an additional benefit for the usage in industry environments. But even though positioning and orientation of the sensor did not seem to have an impact on its performance for the tested flow conditions, explicit movements directly at the LMD nozzle while being mounted there were not investigated yet and have to be considered for future works and a potential roll out in process environments. For this, comparing studies regarding the manufacturing of sample components with the presented approaches are planned. The findings and systems are in general also applicable for other pneumatically conveyed powder processes like coating.

Regarding future research, the general validity of the found dependency between sensor output $S$ and powder mass flow rate $\dot{m}$ is a main aspect. It has to be clarified whether a different feeder line or feeder type has an influence on the fitted function. Furthermore, the 
functionality, adaptability, and operating range of the optical sensor approach can be exceeded. The relation between carrier gas flow rate $\dot{V}$ and the resulting scaling factor $a$ can be determined with more experimental data at different carrier gas flow rates. With this, the in situ measurement of the actual powder mass flow rate by the optical sensor would be possible at variable carrier gas flow rates. In a similar manner, the usage of the optical sensor with other powders than the investigated nickel-based powder is interesting. It would reveal the influence of parameters like particle sizes and densities on the relation between $S$ and $\dot{m}$ while recycled powders might be taken into account as well. With this, a powder that was not investigated before can be directly measured and monitored in situ without any weighing scale recordings.

For the closed-loop controller, similar investigations are possible with two main targets: increase of the range of application and further performance improvements. Both inhere a better understanding of the underlying processes for the non-linear behavior of the vibration feeder. Finding the general dependency of the controller gains on the utilized powder and carrier gas setting would result in a more universal applicability. With changing parameters like powder densities, median particle size $d_{50}$, and carrier gas flow rate $\dot{V}$, the matching controller gains could be determined without or with only a limited number of step response measurements. Other research would include a more detailed view on the steady state with the longterm behavior and possible improvements for this aspect. Furthermore, the usage of the closed-loop control approach on different vibration feeder lines and systems would give information about the transferability. Eventually, a focus could be laid on the configurations in which a lower powder mass flow rate was active before the step. As described in Section 4.2, they show oscillating deviations and are the hardest to control.

Author contribution The corresponding author PP Breese has been responsible for planning and conducting this research along with writing the publication. T Hauser supported in experimental setup and procedure as well as in structuring and review. D Regulin has been responsible for correction, review, and supervision. S Seebauer supported in review and validation. C Rupprecht supported in research coordination and supervision.

Funding Open Access funding enabled and organized by Projekt DEAL.

Data availability All data gathered regarding this publication is presented.

\section{Declarations}

Consent for publication Not applicable as this research did not involve animals or human participants.
Conflict of interest The authors declare no competing interests.

Open Access This article is licensed under a Creative Commons Attribution 4.0 International License, which permits use, sharing, adaptation, distribution and reproduction in any medium or format, as long as you give appropriate credit to the original author(s) and the source, provide a link to the Creative Commons licence, and indicate if changes were made. The images or other third party material in this article are included in the article's Creative Commons licence, unless indicated otherwise in a credit line to the material. If material is not included in the article's Creative Commons licence and your intended use is not permitted by statutory regulation or exceeds the permitted use, you will need to obtain permission directly from the copyright holder. To view a copy of this licence, visit http://creativecommons. org/licenses/by/4.0/.

\section{References}

1. Tan H, Zhang F, Fu X, Meng J, Hu G, Fan W, Huang W (2016) Development of powder flow model of laser solid forming by analysis method. Int J Adv Manuf Technol 82(5-8):1421-1431

2. Gaja H, Liou F (2018) Defect classification of laser metal deposition using logistic regression and artificial neural networks for pattern recognition. Int J Adv Manuf Technol 94(1-4):315-326

3. Tang L, Ruan J, Landers RG, Liou F (2008) Variable powder flow rate control in laser metal deposition processes. J Manuf Sci Eng Trans ASME 130(4):0410161-04101611

4. Mahamood RM, Akinlabi ET (2017) Scanning speed and powder flow rate influence on the properties of laser metal deposition of titanium alloy. Int J Adv Manuf Technol 91(5-8):2419-2426

5. Hauser T, Breese PP, Kamps T, Heinze C, Volpp J, Kaplan AF (2020) Material transitions within multi-material laser deposited intermetallic iron aluminides. Addit Manuf 34

6. Yan Y (1996) Mass flow measurement of bulk solids in pneumatic pipelines. Meas Sci Technol 7(12):1687-1706

7. Nowak S (2016) Three ways to improve continuous loss-in-weight feeding accuracy. Powder Bulk Eng (December)

8. Thayalan V, Landers RG (2006) Regulation of powder mass flow rate in gravity-fed powder feeder systems. J Manuf Process $8(2): 121-132$

9. Li L, Steen WM (2018) Sensing, modelling and closed loop control of powder feeder for laser surface modification 965: 965-974

10. Kahriman A, Ozer U, Karadogan A, Ozdemir K, Tuncer G, Kaya E (2008) Effects of particle size distribution on loading performance. Proc 34th Ann Conf Explos Blasting Tech 1:279-284

11. Liu B, Wildman R, Tuck C, Ashcroft I, Hague R (2011) Investigation the effect of particle size distribution on processing parameters optimisation in selective laser melting process. 22nd Annu Int Solid Free Fabr Symp - An Addit Manuf Conf SFF 2011 (January 2015), pp 227-238

12. Kaleem MA, Alam MZ, Khan M, Jaffery SHI, Rashid B (2020) An experimental investigation on accuracy of Hausner Ratio and Carr Index of powders in additive manufacturing processes. Met Powder Rep (0026-0657)

13. Tripathi NM, Francqui F, Lumay G (2020) Influence of relative air humidity on the flow property of fine powders. In: Third int. Conf. powder, Granule Bulk Solids Innov. Appl. PGBSIA 2020 Febr. 26-28, 2020, p 63

14. Dietrich K, Messe O, Arunprasad T, Foret P, Schoberth A, Szost $\mathrm{B}$, Gerd W (2019) The influence of humidity in Ti-6Al-4V powder on final part quality - Part 2, pp 2-7 
15. Dennis JE, El-Alem M, Williamson K (1999) A trust-region approach to nonlinear systems of equalities and inequalities. SIAM J Optim 9(2):291-315

16. Reisch R, Hauser T, Kamps T, Knoll A (2020) Robot based wire arc additive manufacturing system with context- sensitive multivariate monitoring framework. In: Procedia Manuf., vol 51. Elsevier B.V., pp 732-739

17. Zhang X, Gong W, Xiang H, Chen Y, Li D, Wang Y (2020) Cloud-based AGV control system. In: Lect. Notes Inst. Comput. Sci. Soc. Telecommun. Eng. LNICST, vol 322. Springer Nature, pp 283-293
18. Gross C, Voelker H (2020) A comparison of tuning methods for PID-controllers with fuzzy and neural network controllers. Lect Notes Networks Syst 95:74-86

19. Parandoush P, Hossain A, Yusoff N (2015) Numerical and intelligent analysis of silicon nitride laser grooving. Int $\mathbf{J}$ Adv Manuf Technol 79(9-12):1849-1859

Publisher's note Springer Nature remains neutral with regard to jurisdictional claims in published maps and institutional affiliations. 